\title{
Action-oriented obesity counseling attains weight stabilization and improves liver enzymes among overweight and obese children and adolescents
}

\author{
Allison C. Sylvetsky ${ }^{1}$, Jean A. Welsh ${ }^{2}$, Stephanie M. Walsh ${ }^{2,3}$, Miriam B. Vos $^{2,3}$ \\ ${ }^{1}$ Department of Pediatrics, School of Medicine, Emory University, Atlanta, USA \\ ${ }^{2}$ Nutrition and Health Sciences Program, Division of Biological and Biomedical Sciences, Laney Graduate School, Emory University, \\ Atlanta, USA \\ ${ }^{3}$ Children's Healthcare of Atlanta at Egleston, Atlanta, USA \\ Email: asylvet@emory.edu
}

Received 18 February 2012; revised 28 April 2012; accepted 14 May 2012

\begin{abstract}
Introduction: Pediatricians are encouraged to promote behavior modification to reduce childhood obesity and its co-morbidities, yet the effectiveness of office counseling is unclear. We aimed to evaluate if a low-intensity intervention (action-oriented counseling) in a clinic setting results in weight stabilization, and if the effect is modified by a diagnosis of non-alcoholic fatty liver disease (NAFLD). We hypothesized that patients with NAFLD would be more motivated to adhere to the lifestyle goals set in clinic, due to the diagnosis of an obesity-related condition; and, would therefore achieve greater weight reduction compared to similarly overweight and obese patients without a diagnosis of NAFLD. Methods: A retrospective chart review was conducted on 73 (35 male, 38 female) overweight and obese patients (BMI $\geq 85$ th percentile) attending a pediatric GI clinic between January 2006 and October 2011. Analysis was conducted to determine if lifestyle goals discussed with the patient at each clinic visit were associated with improved BMI, BMI z-score, and liver enzymes. Treatment outcomes among NAFLD patients and similarly obese patients without NAFLD were compared using t-tests and chi-square tests. Results: Of the children evaluated, $\mathbf{7 4 . 0 \%}$ achieved a reduction or stabilization in BMI z-score after 3 months of follow-up. Among NAFLD patients, liver enzymes improved in $72 \%$ of those who were able to stabilize or reduce their BMI and among $43 \%$ of those who gained weight. Treatment outcome did not significantly differ based on having a diagnosis of NAFLD, although there was a trend towards greater improvements. Conclusion: Our study suggests that action oriented counseling including goalsetting in a low intensity, clinic based approach is effective in improving patient BMI, in the presence or absence of an obesity-related co-morbidity, such as
\end{abstract}

NAFLD. Further, we demonstrated that lifestyle modification led to improvement of liver enzymes in NAFLD patients and may result in other clinically relevant improvements. Longer studies will be needed to determine if the improvements are sustained.

Keywords: Obesity; Non-Alcoholic Fatty Liver Disease; Lifestyle; Behavior Change

\section{INTRODUCTION}

The prevalence of childhood obesity has increased dramatically [1] and is associated with type 2 diabetes, cardiovascular disease and cancer, among other chronic diseases [2]. This is alarming because children categorized as overweight and obese often continue on a trajectory of weight gain and remain overweight and obese as adults. The likelihood that the excess weight gain continues into adulthood increases when a child's weight gain persists over a long time period and it is therefore imperative that weight-related behaviors are addressed as early as possible. Because obesity results from a complex interplay of genetic, interpersonal, and environmental factors, pediatricians are urged to work with both patients and their families to encourage behavior change [3]. However, predictors of successful treatment outcomes are inconclusive among obese adults [4] and have not been well-studied in a pediatric population.

Though randomized controlled trials with high intensity interventions have led to successful weight outcomes among children and adolescents $[5,6]$, little data exist to support effective obesity management in routine outpatient clinic settings or to definitively determine the frequency of intervention required. The most recent recommendations from the US Preventive Task Force, suggests that all obese children $>6$ years of age be offered or referred to a moderate to high intensity program involving $>25$ 
hours of contact with the child and/or family over 6 months [7]. Although these multi-faceted and resource intensive weight loss programs, involving weekly nutrition education sessions, homework assignments, group physical activity sessions, and frequent follow-up phone calls, are effective for some [8-10], they are unaffordable and impractical for many others. In many cases, resources are lacking to support multidisciplinary teams consisting of physicians, nurses, dieticians, physical therapists and/or psychologists, as have been utilized in several successful childhood obesity interventions [10-12].

Prior research has demonstrated that increasing knowledge about obesity and its causes has limited effectiveness in achieving lifestyle modification and weight reduction [13], and it has been suggested that motivation is more important than information in facilitating behavior change [14]. In order to successfully and sustainably modify patient behavior, the patient must agree that they need to change their current habits and be motivated to make healthier choices [15]. In utilizing a patient-centered approach to lifestyle modification in our clinic, we work with each patient and their family to elicit behavior change through understanding their perception of their current lifestyle habits, and empowering them to change their environment and ultimately their behaviors, without persuasion or criticism [16]. This patient-centered approach (motivational interviewing), has achieved success in improving weight-related behavior $[17,18]$ and has also shown promise in teen smoking cessation $[19,20]$ and alteration of other risky activities such as alcohol use [21] and unprotected sex [22]. Beyond initial weight loss, motivational interviewing has been effective in sustained lifestyle modification, leading to long-term weight maintenance [23].

The objective of this study was to determine if goaloriented counseling utilizing components of motivational interviewing in relatively brief, infrequent clinic visits at a pediatric gastroenterology practice were effective in stabilizing BMI z-score in a diverse population of overweight children and adolescents. Because our clinic serves both overweight children without co-morbidities and those with complications, we also sought to investigate if having a co-morbidity present enhanced the success of the counseling, possibly due to increased motivation. The most common co-morbidity in the clinic is NAFLD (non-alcoholic fatty liver disease), a chronic, obesity related liver disease. We hypothesized that NAFLD patients would be more responsive to the lifestyle goals set in the clinic due to their clinical diagnosis of an obesity related condition and thus more effective in their weight loss/stabilization efforts.

\section{METHODS}

We conducted a retrospective chart review of overweight and obese patients (BMI $>85$ th percentile) attending the Health 4 Life clinic at Children's Healthcare of Atlanta between January 2006 and October 2011. The Health 4 Life clinic was set up for children referred to GI for obesity and/or obesity with liver enzyme elevation. To meet the pre-determined chart review criteria, patients needed to be 1) referred to and seen in the Health 4 Life clinic; 2) attended $\geq 3$ visits; 3 ) were followed for $>90$ days; and 4) had a primary indication of excess weight gain or excess weight gain and elevated liver enzymes. A total of 231 medical charts were reviewed by a single investigator (AS) uninvolved with the clinic.

All patients were seen at the Children's Healthcare of Atlanta Health 4 Life clinic after referral by their primary care physician for concerns relating to excess weight gain or elevated liver enzymes along with excess weight gain. We chose to study NAFLD because it is the most common co-morbidity of obesity seen in our clinic; whereas other gastrointestinal issues found in overweight children (i.e. reflux, constipation), are not caused by obesity. Each patient was seen by a pediatric gastroenterologist (MBV) and a nutritionist, both of whom were experienced in and consistently used patient centered action-oriented counseling, a method shown to be effective in modifying obesity-related behaviors [24].

Action-oriented strategies are based on the Stages of Change Model [25], which builds upon on the patient's perceptions of their current behaviors and their motivation and intention to change [26]. Specifically, the clinic visits followed the 5A's format [24] (ask, advise, assess, assist arrange), which is depicted in Table 1 and has previously been shown to be effective in motivating obese patients to lose weight [27]. Typical visits lasted $\sim 45$ minutes and included collecting information about

Table 1. Description of each of the " 5 A's" used in behavior change counseling in the Health 4 Life clinic.

\footnotetext{
The "ask" stage involves asking the patient and their family about their current lifestyle habits and evaluating

ASK their willingness to change. Willingness to change is determined by asking the family to complete a questionnaire, either verbally or on paper.

The "advise" stage involves discussing best practice recommendations for the lifestyle habits discussed in the previous stage.

We then "assess" what the patient and their family

ASSESS would like to change, and what they perceive as facilitators and barriers of successfully making the changes discussed.

We then "arrange" for a follow-up visit, at which point ARRANGE we will monitor the patient's progress by repeating each the $5 \mathrm{~A}$ 's.
}

${ }^{1}$ The "5 A's" used in our Health 4 Life clinic are adapted from Joy et al. 2008 . 
health habits, assessment of the family's willingness to change and their priorities for behavior change, and provider facilitated goal setting. Patients and their families were asked about their current lifestyle, provided age appropriate lifestyle recommendations targeted to their situation, assessed for preference of area to work on and barriers and then assisted in setting a practical, achievable goal based on their responses. Because the clinic was a referral clinic and most families were referred for weight related concerns, they were generally already motivated. At the end of each of the visits, two goals were chosen by the family: one related to nutrition and one to physical activity. Examples of goals set included "limit TV to one hour a day on school days", "go to the park for 30 minutes 3 times a week", or "limit sugar drinks to 2 times a week".

Most patients were seen 1 month after their initial visit and then 2 - 6 months later. During follow-up visits, the lifestyle goals set at the previous visit were discussed, and with the pediatrician's guidance, patients decided which goals they wanted to continue to pursue. For the purpose of this study, we defined the 3-month visit as the visit closest to 90 days and the 6-month visit as the visit closest to 180 days after the initial visit. Due to cancelations, variable patient progress and need to follow up patient liver enzymes elevations, there was considerable variability in the time between visit intervals, as displayed in Table 2.

Information was extracted from dictated notes in the patient medical charts. Height, weight, BMI, BMI z-score, and date of birth were recorded for each patient at the baseline visit. Among those referred to the clinic for concerns regarding NAFLD, ALT and AST values were also extracted. Since BMI changes naturally as children grow, we used patients' BMI z-scores (a measure of a child's weight relative to a reference population) calculated using the CDC 2000 Growth Charts as the reference [28] to monitor the weight trend of our patients [29]. A trend that demonstrated stabilization or decrease in BMI

Table 2. Variability (months) between initial visit and followup visit "closest to 3 months" and "closest to 6 months" for all patients, NAFLD patients, and non-NAFLD patients.

\begin{tabular}{cccc}
\hline & All & NAFLD & Non-NAFLD \\
& \multicolumn{2}{c}{ Visit closest to 3 months } & \\
\hline $\mathbf{n}$ & 73 & 25 & 48 \\
$\begin{array}{c}\text { Median } \\
\text { (25th, 75th) }\end{array}$ & 4.30 & 4.80 & 4.2 \\
\hline & $(3.73,5.45)$ & $(3.82,5.65)$ & $(3.73,5.13)$ \\
\hline All & NAFLD & Non-NAFLD \\
V & Visit closest to 6 months & \\
Median & 56 & 22 & 34 \\
(25th, 75th) & $(7.00,9.86)$ & $(7.24,11.20)$ & $(7.00,8.93)$ \\
\hline
\end{tabular}

z-score was considered a positive outcome.

Each patient's BMI z-score measured at baseline was compared to the BMI z-score measured at the 3 month and 6-month visits. Weight stabilization was defined as a BMI z-score that was within \pm 0.04 units of the baseline Weight reduction was classified as a BMI z-score decrease of $\geq 0.041$, corresponding to a reduction of greater than $2 \%$. This has been previously demonstrated be a clinically relevant reduction and lead to improvements in metabolic risk factors including trigylcerides, low-density lipoprotein, body composition, and insulin sensitivity [30]. Percent change in AST and ALT between each patient's first and final visit to our clinic was calculated to assess change in liver function over the treatment period.

The Institutional Review Boards at Children's Healthcare of Atlanta and at Emory University approved the study protocol and the extraction of de-identified patient data for use in our study. Patient consent was not obtained because we extracted only de-identified data and because collecting consent from patients seen several years ago would have been prohibitive, given our study design.

All statistics were performed in SAS 9.2 (SAS Institute, Cary, NC) and Microsoft Excel. Results are reported as mean (standard error). Mean change in BMI and BMI z-score between the baseline visit and 3-month and 6-month visits, respectively, were compared using ANOVA. $x^{2}$ tests were used to compare proportions of patients whose BMI z-score increased, decreased, or stabilized between groups. All p-values two-sided and were considered statistically significant if $<0.05$.

\section{RESULTS}

Seventy-three overweight children met the inclusion criteria for our study, 25 of whom were diagnosed with NAFLD and 48 of whom had no prior NAFLD diagnosis. Characteristics of our sample at baseline are shown in Table 3.

Seventy-four percent of the patients had main tained or decreased their BMI z-score at the 3-month visit, 72.0\% $(\mathrm{n}=18)$ and $75.3 \%(\mathrm{n}=36)$ in the NAFLD and non-NAFLD groups, respectively. The proportions of

Table 3. Characteristics of the sample at baseline.

\begin{tabular}{ccccc}
\hline & All & NAFLD & Non-NAFLD & $\mathrm{p}$ \\
\hline $\mathrm{N}$ & 73 & 25 & 48 & \\
Male (\%) & $35(48)$ & $18(72)$ & $19(40 \%)$ & $\mathrm{p}=0.01$ \\
Female (\%) & $38(52)$ & $7(28)$ & $28(60 \%)$ & \\
Age (years) $^{2}$ & $12.47(0.35)$ & $12.21(0.49)$ & 12.61 & $\mathrm{NS}^{1}$ \\
BMI z-score $^{2}$ & $2.52(0.05)$ & $2.45(0.06)$ & $2.56(0.07)$ & $\mathrm{NS}$ \\
\hline
\end{tabular}

${ }^{1}$ Not statistically significant at $\mathrm{p}<0.05 ;{ }^{2} \mathrm{Age}$, BMI z-score, and liver enzymes are presented as mean (standard error). 
children whose BMI z-score stabilized, increased, and decreased from baseline to the 3-month visit are shown in Figure 1. Among those with NAFLD 72\% $(n=13)$ of patients who demonstrated a favorable weight trajectory at 3 months improved their ALT values, compared to only $43 \%$ of those children who continued to gain weight. Mean AST values decreased by $8.4 \%$, from $73 \pm 7 \mathrm{U} / \mathrm{L}$ at the initial visit, to $61 \pm 9 \mathrm{U} / \mathrm{L}$ at the final visit but were not statistically significant. Mean ALT values decreased by $25.6 \%$, from $105 \pm 9$ at the initial visit to $80 \pm 13$ at the subsequent visit and this was statistically significant $(\mathrm{p}=0.02)$.

Over $75 \%$ of the patients returned for the 6-month visit, $88 \%(\mathrm{n}=22)$ in the NAFLD group and $70.8 \%$ in the non-NAFLD group $(n=34)$. Follow-up at 6 months was not statistically different based on gender, NAFLD status, or weight trend at 3 months. The proportions of children whose BMI z-score stabilized, increased, or decreased at 6 months are shown in Figure 2. There was no difference in the percentage of patients with a positive

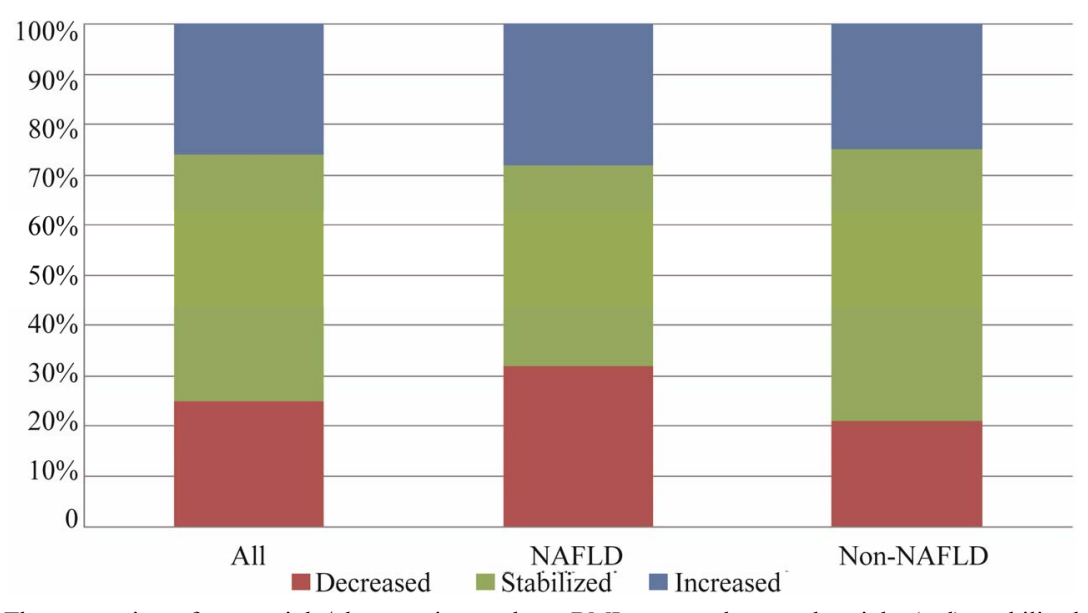

The proportion of overweight/obese patients whose BMI z-score decreased weight (red), stabilized (green), and increased (blue) at the 3 month follow-up clinic visit compared to baseline in the total sample (left), NAFLD patients (center), and non-NAFLD patients (right). A cutoff of \pm 0.04 was used to define stabilization for the purpose of this study and a reduction of more than $0.04 \mathrm{z}$-score was considered weight loss. ${ }^{1}$ Proportion of patients who decreased, stabilized, and increased their BMI z-score at 3 month follow-up visit was not statistically different between the NAFLD and non-NAFLD patients $(\mathrm{p}=0.45)$.

Figure 1. Proportion of overweight/obese patients ${ }^{1}$ whose BMI decreased, stabilized, or increased after 3 months of follow-up.

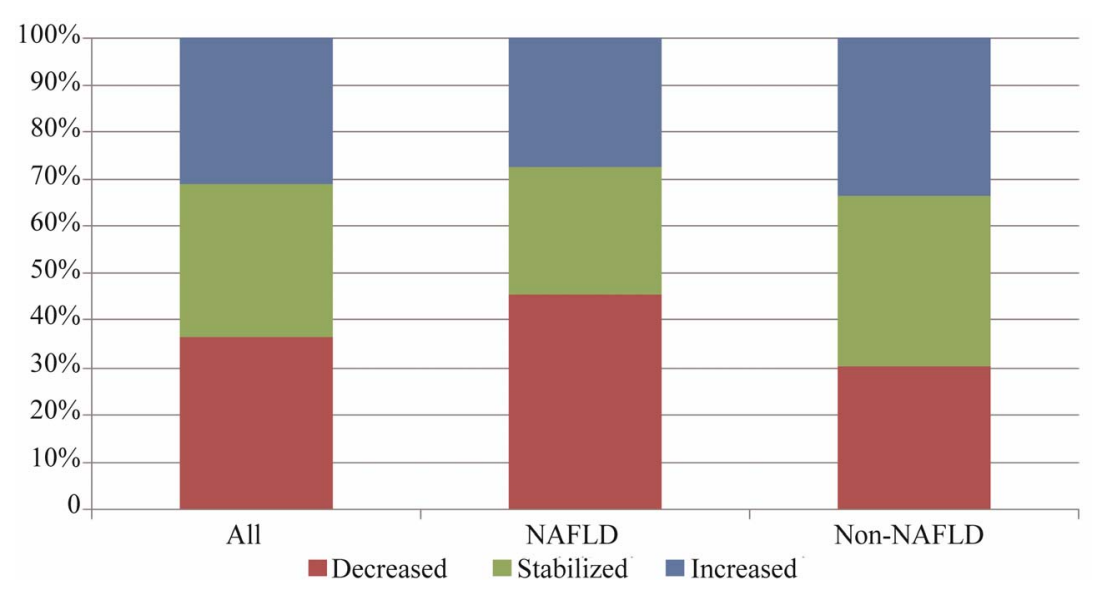

The proportion of overweight/obese patients whose BMI z-score decreased weight (red), stabilized (green), and increased (blue) at the 3 month follow-up clinic visit compared to baseline in the total sample (left), NAFLD patients (center), and non-NAFLD patients (right). A cutoff of \pm 0.04 was used to define stabilization for the purpose of this study and a reduction of more than $0.04 \mathrm{z}$-score was considered weight loss. ${ }^{2}$ Proportion of patients who decreased, stabilized, and increased their BMI z-score at the 6 month follow-up visit was not statistically different between the NAFLD and non-NAFLD patients $(\mathrm{p}=0.51)$.

Figure 2. Proportion of overweight/obese patients ${ }^{2}$ whose BMI decreased, stabilized, or increased after 6 months of follow-up. 
outcome (stabilization or reduction) when comparing patients with and without NAFLD.

\section{DISCUSSION}

Contrary to our hypothesis, we found that treatment outcomes were positive in both patients with and without a diagnosis of NAFLD. This was surprising because we expected that those previously diagnosed with NAFLD would be more motivated to change and would therefore adhere more closely to the lifestyle goals discussed in clinic. To our knowledge, this is the first study to compare weight reduction outcomes between children with and without an obesity-associated chronic disease in a clinic setting. Various studies have examined predictors of treatment success and follow-up in weight loss clinics [31-33], yet most have examined demographic factors rather than weight-related health conditions. Thus, the association between overt pathological conditions resulting from obesity and motivation to comply with lifestyle change recommendations has not been well-studied. Though our results suggest that children and adolescents with NAFLD do not respond to treatment differently than similarly obese children without a diagnosed comorbidity, this concept warrants further investigation.

Our study was designed to evaluate whether a low intensity approach utilizing office-based, one-on-one visits with a pediatric gastroenterologist and nutritionist were effective in promoting weight stabilization, and to examine if treatment outcomes differed between overweight children with and without NAFLD. The key component promoting behavior change was the use of patient centered, action-oriented counseling techniques. With these strategies, the patient-provider relationship has been shown to develop quickly and improve the intentions of patients to change their behaviors.

The results of our study suggest that counseling was effective in short-term stabilization of BMI and in improving liver enzymes in our clinic. The proportion of our patients who achieved stabilization or reduction BMI $\mathrm{z}$-score was similar to success rates in other clinic-based weight management programs [34]. Liver enzymes improved in $72 \%$ of NAFLD patients who reduced or stabilized their BMI z-score and in $43 \%$ of those whose BMI $z$-score increased, emphasizing the importance of lifestyle behavior change, independent of weight reduction. Though most patients remained overweight or obese at the 3-month and 6-month follow-up visits, our findings suggest that lifestyle change, even in the absence of weight loss, leads to metabolic improvement. This finding is supported by previous research where improvements liver enzymes were achieved with lifestyle change, independent of weight reduction or with minimal weight change [35-37].

Our Health 4 Life clinic was set up to treat patients with elevated liver enzymes and/or excess weight gain, and hence was based on a model of low intensity intervention with patient visits $3-4$ times in the over the first year. Though our patients were seen in a sub-speciality clinic, our office-based counseling was less resource and time intensive than what is typically found in a most formal obesity treatment programs. We did have the added benefit of a nutritionist in addition to the clinician, and given improved insurance support for nutrition visits in primary care offices, our model could be replicated in a general pediatric office as well as other pediatric gastroenterology offices. As such, our findings are encouraging for reduction of weight gain among obese patients seen in both non-specialty and specialty clinics. Actionoriented counseling by a different trained professional, such as a medical assistant or licensed practical nurse could also be a cost-effective approach for clinic based programs.

The main limitations of our study were its retrospecttive nature and the high rate of attrition in our clinic, which was comparable to drop-out rates reported in similar pediatric clinics [38-40]. Attrition in pediatric weight management clinics presents a challenge to BMI z-score reduction and sustained lifestyle change, and has been reported to range from $27 \%$ and $73 \%$ [41]. Those patients who failed to return for follow-up may have also stabilized or decreased their BMI; in several prior studies, patients lost to follow-up did not differ in treatment outcome compared to those who returned to the clinic $[39,42]$. Meanwhile, other pediatric studies have demonstrated that patients who adhere to the treatment program are more likely to achieve weight reduction $[43,44]$, though outcome information is largely unavailable among patients lost to follow-up in clinic-based initiatives.

Prior research has suggested that severely obese patients are less likely to return for follow-up, and given that the mean BMI z-score at baseline was more than 2.5 standard deviations above the mean, this may explain the high rate of attrition in our clinic [39]. Other predictors of drop-out including black race, being of lower socioeconomic status [45], being an older adolescents, and expressing low perceived quality of care [46], may also have contributed to loss to follow-up among our patients, though consistent predictors of attrition have not been identified in weight clinics [4].

The large range of follow-up periods that were considered the "closest visit after 90 and 180 days was also a limiting factor." Depending on a patient's specific condition and progress, follow-up visits were scheduled at different intervals. This is typical of most clinics and makes the study relevant to practicing physicians. Also, we were unable to extract information about parental BMI, race/ethnicity, socio-economic status, self-esteem, or prior eating behaviors, as this information was not 
available in the medical charts, which were not designed for research and may have confounded the observed association between participation in action-oriented counseling and weight stabilization. Importantly, we were also unable to obtain the patient's BMI z-score at the time of referral, which may have predicted patient outcomes in our Health 4 Life clinic.

It has been reported that pediatricians find it difficult to effectively promote obesity reduction among their patients. Pediatricians often believe that they are not well-positioned to target weight control in their offices, due to a lack of resources and other restrictions such as reimbursement, billing, and lack of time [47]. The above mentioned barriers coupled with low perceived patient and family motivation to change [48], low enthusiasm for weight management [49] and hesitation to address a sensitive issue [47] among pediatricians, may contribute to the perceived inadequate counseling for childhood overweight in primary care. Parents, specifically those who are overweight, can view weight-related advice provided in the pediatricians' office as inadequate [50], reinforcing the necessity of targeting patient motivation and improving action-oriented counseling abilities among general practitioners.

In contrast, our clinic which was designed for pediatric weight counseling and used patient centered counseling with components of motivational interviewing, did not find these to be barriers and worked with families to build enthusiasm about healthy lifestyles. Our patients and their families generally viewed the experience as positive, and this was supported by the rapport established with the clinician and the dietician [51] and the boosting of patient self-esteem through focusing on successfully implemented behavior change [52], both which have previously been shown to increase parent satisfaction. Our success lends some possible conclusions although further research will be needed to make firm conclusions. In demonstrating that clinic based patient centered counseling can be successful in stabilizing the weight status of overweight/obese patients who return for followup clinic visits and also improves liver enzymes among those with NAFLD, our findings further support the ability of clinicians and nutritionists to provide effective obesity counseling in the outpatient clinic setting and not simply to rely on referral to high intensity, high resource obesity programs. Second, we think that our study supports the utilization of patient centered, action oriented counseling combined with access to nutritionists. Both of these are possible key contributors to our positive results and should be evaluated further, particularly in the primary care setting.

\section{ACKNOWLEDGEMENTS}

This project was supported in part by a grant from NIH/NIDDK
K23DK080953 (Vos) and by support from Strong4Life@ Children's Healthcare of Atlanta.

\section{REFERENCES}

[1] WHO (2009) Population-based prevention strategies for childhood obesity. Report of the WHO Forum and Technical Meeting, WHO, Geneva.

[2] Biro, F.M. and Wien, M. (2010) Childhood obesity and adult morbidites. American Journal of Clinical Nutrition, 91, 1499S-1505S. doi:10.3945/ajcn.2010.28701B

[3] Barlow, S.E. and the Expert Committee (2007) Expert committee recommendations regarding the prevention, assessment, and treatment of child and adolescent overweight and obesity: Summary report. Pediatrics, 120 , S164-S192. doi:10.1542/peds.2007-2329C

[4] Moroshko, I., Brennan, L. and O’Brien, P. (2011) Predictors of dropout in weight loss interventions: A systematic review of the literature. Obesity Reviews, 12, 912934. doi:10.1111/j.1467-789X.2011.00915.x

[5] Waters, E., et al. (2011) Interventions for preventing obesity in children. Cochrane Database of Systematic Reviews, 12, CD001871.

[6] Kesten, J.M., Griffiths, P.L. and Cameron, N. (2011) A systematic review to determine the effectiveness of interventions designed to prevent overweight and obesity in pre-adolescent girls. Obesity Reviews, 12, 997-1021. doi:10.1111/j.1467-789X.2011.00919.X

[7] Barton, M. (2010) Screening for obesity in children and adolescents: US Preventive Services Task Force recommendation statement. Pediatrics, 125, 361-367. doi:10.1542/peds.2009-2037

[8] Jones, R.A., et al. (2007) The HIKCUPS trial: A multisite randomized controlled trial of a combined physical activity skill-development and dietary modification program in overweight and obese children. BMC Public Health, 7, 15. doi:10.1186/1471-2458-7-15

[9] Pinard, C.A., et al. (2012) Smart choices for healthy families: A pilot study for the treatment of childhood obesity in low-income families. Health, Education and Behavior, 39, 433-445. doi:10.1177/1090198111425686

[10] Vignolo, M., et al. (2008) Five-year follow-up of a cognitive-behavioural lifestyle multidisciplinary programme for childhood obesity outpatient treatment. European Journal of Clinical Nutrition, 62, 1047-1057. doi:10.1038/sj.ejen.1602819

[11] Shalitin, S., et al. (2009) Effects of a twelve-week randomized intervention of exercise and/or diet on weight loss and weight maintenance, and other metabolic parameters in obese preadolescent children. Hormone Research, 72, 287-301. doi:10.1159/000245931

[12] Nemet, D., et al. (2005) Short- and long-term beneficial effects of a combined dietary-behavioral-physical activity intervention for the treatment of childhood obesity. Pediatrics, 115, e443-e449. doi:10.1542/peds.2004-2172

[13] Sealy, Y.M. and Farmer, G.L. (2011) Parents' stage of 
change for diet and physical activity: Influence on childhood obesity. Social Work Health Care, 50, 274-291. doi:10.1080/00981389.2010.529384

[14] Resnicow, K., Davis, R. and Rollnick, S. (2006) Motivational interviewing for pediatric obesity: Conceptual issues and evidence review. Journal of the American Dietetic Association, 106, 2024-2033. doi:10.1016/j.jada.2006.09.015

[15] Tripp, S.B., et al. (2011) Providers as weight coaches: Using practice guides and motivational interview to treat obesity in the pediatric office. Journal of Pediatric Nursing, 26, 474-479. doi:10.1016/j.pedn.2011.07.009

[16] Schwartz, R.P. (2010) Motivational interviewing (patientcentered counseling) to address childhood obesity. Pediatric Annals, 39, 154-158. doi:10.3928/00904481-20100223-06

[17] Taveras, E.M., et al. (2011) Randomized controlled trial to improve primary care to prevent and manage childhood obesity: The high five for kids study. Archives of Pediatrics and Adolescent Medicine, 165, 714-722. doi:10.1001/archpediatrics.2011.44

[18] Armstrong, M.J., et al. (2011) Motivational interviewing to improve weight loss in overweight and/or obese patients: A systematic review and meta-analysis of randomized controlled trials. Obesity Reviews, 12, 709-723.

[19] Colby, S.M., et al. (2005) Brief motivational intervention for adolescent smokers in medical settings. Addictive Behaviors, 30, 865-874. doi:10.1016/j.addbeh.2004.10.001

[20] Colby, S.M., et al. (2012) Enhanced motivational interviewing versus brief advice for adolescent smoking cessation: Results from a randomized clinical trial. Addictive Behaviors, 37, 817-823. doi:10.1016/j.addbeh.2012.03.011

[21] Feldstein, S.W. and Forcehimes, A.A. (2007) Motivational interviewing with underage college drinkers: A preliminary look at the role of empathy and alliance. The American Journal of Drug and Alcohol Abuse, 33, 737746. doi:10.1080/00952990701522690

[22] Dermen, K.H. and Thomas, S.N. (2011) Randomized controlled trial of brief interventions to reduce college students' drinking and risky sex. Psychology of Addictive Behaviors, 25, 583-594. doi:10.1037/a0025472

[23] DiLillo, V. and West, D.S. (2011) Motivational interviewing for weight loss. Psychiatric Clinics of North America, 34, 861-869. doi:10.1016/j.psc.2011.08.003

[24] Joy, E.A. (2008) Practical approaches to office-based physical activity promotion for children and adolescents. Current Sports Medicine Reports, 7, 367-372.

[25] Prochaska, J.O. and Velicer, W.F. (1997) The transtheoretical model of health behavior change. American Journal of Health Promotion, 12, 38-48. doi:10.4278/0890-1171-12.1.38

[26] Prochaska, J.O. and DiClemente, C.C. (1992) Stages of change in the modification of problem behaviors. Progress in Behavior Modification, 28, 183-218.

[27] Jay, M., et al. (2010) Physicians' use of the 5As in counseling obese patients: Is the quality of counseling associ- ated with patients' motivation and intention to lose weight? BMC Health Services Research, 10, 159. doi:10.1186/1472-6963-10-159

[28] National Center for Health Statistics (2000) CDC growth charts. http://www.cdc.gov/growthcharts/

[29] Whitlock, E.P., et al. (2005) Screening and interventions for childhood overweight: A summary of evidence for the US Preventive Services Task Force. Pediatrics, 116, e125-e144. doi:10.1542/peds.2005-0242

[30] Ford, A.L., et al. (2010) What reduction in BMI SDS is required in obese adolescents to improve body composition and cardiometabolic health? Archives of Disease in Childhood, 95, 256-261. doi:10.1136/adc. 2009.165340

[31] Porter, J.S., et al. (2010) Psychosocial factors and perspectives on weight gain and barriers to weight loss among adolescents enrolled in obesity treatment. Journal of Clinical Psychology in Medical Settings, 17, 98-102. doi:10.1007/s10880-010-9186-3

[32] Jelalian, E., et al. (2008) Predictors of attrition and weight loss in an adolescent weight control program. Obesity, 16, 1318-1323. doi:10.1038/oby.2008.51

[33] Tershakovec, A.M. and Kuppler, K. (2003) Ethnicity, insurance type, and follow-up in a pediatric weight management program. Obesity Research, 11, 17-20. doi:10.1038/oby.2003.4

[34] Sabin, M.A., et al. (2007) Which factors are associated with a successful outcome in a weight management programme for obese children? Journal of Evaluation in Clinical Practice, 13, 364-368. doi:10.1111/j.1365-2753.2006.00706.x

[35] Reinehr, T., et al. (2009) Lifestyle intervention in obese children with non-alcoholic fatty liver disease: 2-year follow-up study. Archives of Disease in Childhood, 94, 437-442. doi:10.1136/adc.2008.143594

[36] St. George, A., et al. (2009) Effect of a lifestyle intervention in patients with abnormal liver enzymes and metabolic risk factors. Journal of Gastroenterology and Hepatology, 24, 399-407. doi:10.1111/j.1440-1746.2008.05694.x

[37] Koot, B.G., et al. (2011) Lifestyle intervention for non-alcoholic fatty liver disease: Prospective cohort study of its efficacy and factors related to improvement. Archives of Disease in Childhood, 96, 669-674. doi:10.1136/adc.2010.199760

[38] Skelton, J.A., DeMattia, L.G. and Flores, G. (2008) A pediatric weight management program for high-risk populations: A preliminary analysis. Obesity, 16, 1698-1701. doi: $10.1038 /$ oby. 2008.243

[39] Barlow, S.E. and Ohlemeyer, C.L. (2006) Parent reasons for nonreturn to a pediatric weight management program. Clinical Pediatrics, 45, 355-360. doi: $10.1177 / 000992280604500408$

[40] Dalton, W.T. 3rd, et al. (2010) Family functioning and children's response to primary care treatment for overweight: A preliminary study. Journal of Pediatric Nursing, 25, 282-288. doi:10.1016/j.pedn.2009.04.005

[41] Skelton, J.A. and Beech, B.M. (2011) Attrition in paedi- 
atric weight management: A review of the literature and new directions. Obesity Reviews, 12, e273-e281. doi:10.1111/j.1467-789X.2010.00803.X

[42] Madsen, K.A., et al. (2009) A clinic-based lifestyle intervention for pediatric obesity: Efficacy and behavioral and biochemical predictors of response. Journal of Pediatric Endocrinology and Metabolism, 22, 805-814. doi:10.1515/JPEM.2009.22.9.805

[43] Williamson, D.A., et al. (2010) Early behavioral adherence predicts short and long-term weight loss in the POUNDS LOST study. Journal of Behavioral Medicine, 33, 305-314. doi:10.1007/s10865-010-9253-0

[44] Dreimane, D., et al. (2007) Feasibility of a hospital-based, family-centered intervention to reduce weight gain in overweight children and adolescents. Diabetes Research and Clinical Practice, 75, 159-168. doi:10.1016/j.diabres.2006.05.017

[45] Zeller, M., et al. (2004) Predictors of attrition from a pediatric weight management program. The Journal of Pediatrics, 144, 466-470. doi:10.1016/j.jpeds.2003.12.031

[46] Cote, M.P., et al. (2004) Service quality and attrition: An examination of a pediatric obesity program. International Journal of Quality in Health Care, 16, 165-173. doi:10.1093/intqhc/mzh015
[47] Walker, O., et al. (2007) A qualitative study of primary care clinicians' views of treating childhood obesity. BMC Family Practice, 8, 50. doi:10.1186/1471-2296-8-50

[48] Holt, N., et al. (2011) Primary care practice addressing child overweight and obesity: A survey of primary care physicians at four clinics in southern Appalachia. Southern Medical Journal, 104, 14-19. doi:10.1097/SMJ.0b013e3181fc968a

[49] Mercer, S.W. and Tessier, S. (2001) A qualitative study of general practitioners' and practice nurses' attitudes to obesity management in primary care. Health Bulletin, 59, 248-253.

[50] Taveras, E.M., et al. (2008) Parental perceptions of overweight counseling in primary care: The roles of race/ethnicity and parent overweight. Obesity, 16, 1794-1801. doi:10.1038/oby.2008.264

[51] Stewart, L., et al. (2008) The use of behavioural change techniques in the treatment of paediatric obesity: Qualitative evaluation of parental perspectives on treatment. Journal of Human Nutrition and Dietetics, 21, 464-473. doi:10.1111/j.1365-277X.2008.00888.x

[52] Stewart, L., et al. (2008) Parents' journey through treatment for their child's obesity: A qualitative study. Archives of Diseases in Childhood, 93, 35-39. $\underline{\text { doi:10.1136/adc.2007.125146 }}$ 